\title{
Lungenultraschall bei COVID-19-Patienten ist auf Intensivstation das Mittel der Wahl
}

Rund 7,5\% aller COVID-19-Erkrankten werden intensivmedizinisch behandelt. Grund dafür ist meist die Lungenentzündung, die durch das neuartige Coronavirus ausgelöst wird. Sie kommt schleichend, bleibt lange unbemerkt, kann dann aber innerhalb von wenigen Stunden zu schweren Atemproblemen führen, die eine sofortige Behandlung auf der Intensivstation erfordern. Die Erfahrungen der vergangenen Wochen zeigen, dass dann der Lungenultraschall ein unverzichtbares Überwachungsinstrument für die kontinuierliche und schnelle Verlaufskontrolle der Schwerkranken darstellt. Die Thorax-Sonografie ist unkompliziert, strahlungsfrei, direkt am Intensivbett einsetzbar und kann beliebig oft wiederholt werden. Welche Vorteile sich dadurch für die Patienten ergeben, haben Experten der Deutschen Gesellschaft für Ultraschall in der Medizin e. V. (DEGUM) bei einer Online-Pressekonferenz Ende Mai beantwortet.

Bei rund 7,5\% [1] aller Corona-Infizierten in Deutschland verläuft die COVID-19-Erkrankung so schwer, dass sie intensivmedizi- nisch behandelt werden müssen. Zehn Tage dauert es im Schnitt vom Beginn der ersten Symptome bis zu einer Einweisung auf die Intensivstation. Das Tückische: Der Zustand der betroffenen Patienten kann sich in kurzer Zeit rapide verschlechtern. „Das Lungenversagen tritt innerhalb weniger Stunden auf, sodass eine Intubation bevorsteht", erklärt Professor Dr. med. Dirk-André Clevert. Der Oberarzt an der Klinik und Poliklinik für Radiologie und Leiter des Interdisziplinären Ultraschall-Zentrums im Klinikum der Universität MünchenGroßhadern hat in den vergangenen Monaten fast täglich Patienten mit schwerer COVID-19-Lungenentzündung behandelt. Die Betroffenen können zum Teil zwischen wenigen Tagen und mehreren Wochen auf der Intensivstation verweilen. „Die Veränderungen der Lunge müssen bei den Intensivpatienten regelmäßig überprüft werden“, erklärt Professor Dr. med. Josef Menzel, Neupräsident der DEGUM. „Neben der klinischen Überwachung bedarf es einer regelmäßigen Bildgebung, um das Krankheitsbild besser zu beurteilen und rechtzeitig die richtigen Schritte einzuleiten."
Auf der Intensivstation kann nur der konventionelle Röntgen-Thorax angeboten werden oder der Lungenultraschall - die Computertomografie (CT) steht in der Regel auf den Intensivstationen nicht zur Verfügung. „Somit müssten die schwerstkranken Patienten mit aufwendigen Lagerungs- und Transportmaßnahmen zum CT gebracht werden“, erläutert Clevert, der bei der DEGUM die Sektion Radiologie leitet. Da sowohl der Transport als auch die Umlagerung für diese sehr instabilen Patienten unzumutbar seien, könne zumeist auf eine CT-Bildgebung nicht zurückgegriffen werden. „Der Ultraschall sichert in diesen Fällen die kontinuierliche und schnelle Verlaufskontrolle.“

Die Thorax-Sonografie ist unkompliziert, direkt am Intensivbett einsetzbar und kann beliebig oft wiederholt werden. Im Gegensatz zum Röntgen ist der Ultraschall strahlungsfrei „Die typischen peripheren Verdichtungen, die bei einer COVID-19-Infektion auftreten, lassen sich zuverlässig erfassen und im Verlauf sonografisch beurteilen", so Clevert. Erfahrungen der vergangenen Wochen zeigen, dass die Veränderungen der Lunge mit einem Ultraschall fast genauso 
gut erkennbar seien wie im CT. „Das Zusammenspiel aus der klinischen Symptomatik und den erfassten sonografischen Pathologien ermöglicht eine verbesserte Einschätzung über den Verlauf der Erkrankung. Bei
Bedarf kann die Sonografie auch bei Interventionen, zum Beispiel zur Anlage von Pleuradrainagen oder zentralen Gefäßzugängen, am Intensivbett eingesetzt werden“, so der DEGUM-Experte abschließend.

\section{Literatur}

[1] https://de.statista.com/statistik/daten/studie/ 1108578/umfrage/intensivmedizinischeversorgung-von-corona-patienten-covid-19in-deutschland/ 